\title{
La evaluación por competencias en la docencia universitaria del Grado en Bellas Artes
}

\author{
María del Carmen Bellido MÁrQueZ \\ Universidad de Granada (España) \\ cbellido@ugr.es
}

\begin{abstract}
Resumen
La nueva enseñanza universitaria enmarcada en el Espacio Europeo de Educación Superior está planteada para que el alumno desarrolle competencias (actitudes, capacidades y habilidades) que le preparen para su acceso al mundo laboral. Derivado de estos nuevos planteamientos, el sistema de evaluación requiere una profunda renovación. Por ello, esta investigación está dedicada el estudio y desarrollo de la evaluación por competencias en el Grado en Bellas Artes, aplicada en la asignatura Escultura III: Metodologías Escultóricas (2012-2013), y a la mejora docente que ha generado su aplicación.
\end{abstract}

Palabras claves: Docencia universitaria. Evaluación por competencias. Innovación docente.

The evaluation by competences in university teaching of degree in fine arts

\begin{abstract}
The new university framed in the European Higher Education Space is designed for students to develop competencies (attitudes, skills and abilities) that this will prepare for their access to employment. Derived from these new approaches, the evaluation system requires a thorough renovation. Therefore, this research is dedicated the study and development of evaluation system by competences in the Degree of Fine Arts, applied in the subject Sculpture III: Methodologies Sculptural (2012-2013), and teaching improvement generated by your application.
\end{abstract}

Key words:University teaching. Evaluation by competences. Teaching innovation.

\section{Referencia normalizada:}

Bellido Márquez, M. C. (2013) La evaluación por competencias en la docencia universitaria del Grado en Bellas Artes. Historia y Comunicación Social. Vol. 18. N Especial Diciembre. Págs. 865-877.

Sumario: 1. Introducción. 2. Antecedentes. 3. Objetivos. 4. Metodología. 5. Cronograma. 6 Resultados. 6.1. Estudio de las competencias. 6.2. Evaluación de las competencias. 6.2.1. Construcción de técnicas e instrumentos para la evaluación de la adquisición de competencias y elaboración del sistema de calificación. 6.2.2. Procedimiento de evaluación. 6.2.3. Resultados de evaluación. 7 Conclusiones. 8. Referencias bibliográficas.

\section{Introducción}

El nuevo Espacio Europeo de Educación Superior (EEES) ha sido diseñado para el desarrollo por los estudiantes de competencias basadas en actitudes, capacidades y habilidades que le permitan su integración en el mundo laboral. Desde esta perspectiva, los cambios en la innovación docente son obligados, pues en la evaluación del 
alumno ha de modificarse la sola consideración de la ampliación de sus conocimientos (planes docentes antiguos) por la valoración de la adquisición de las competencias que especifique su titulación, como es inmanente al Plan de Bolonia, (Ibarra y Rodríguez, 2010), que hagan que sea capaz de superar las materias propias de la misma y que optimicen su formación, dotándole de útiles que le ayuden en su inclusión en el mundo laboral, para generar un rendimiento profesional óptimo (Álvarez, 2010).

En el nuevo Plan Bolonia de Estudios Universitarios la competencia está definida como:

\begin{abstract}
"Por competencia se entiende el conjunto de conocimientos, habilidades, actitudes que se adquieren o desarrollan mediante experiencias formativas coordinadas, las cuales tienen el propósito de lograr conocimientos funcionales que den respuesta de modo eficiente a una tarea o problema de la vida cotidiana y profesional que requiera un proceso de enseñanza y aprendizaje" (ANECA, 2012).
\end{abstract}

Dada la generalidad de la definición de competencia, al analizar su contenido resulta complejo y difícil de adquirir. Se necesita estudiar los contenidos de dichas competencias y proceder a su segregación para poder identificarlos y lograrlos de forma progresiva durante el proceso de enseñanza.

Este trabajo de investigación versa sobre el estudio y desarrollo de tres competencias generales del Grado en Bellas Artes (Universidad de Granada) y tres específicas, contempladas en la asignatura Escultura III: Metodologías escultóricas, según está incluido en su Guía Docente (UGR, 2012). Ha sido desarrollada durante el curso académico 2012-2013, en los Grupos B (horario de mañana) y D (horario de tarde), y ha consistido en la aplicación de mecanismos de formación orientados a la adquisición de dichas competencias por los estudiantes, así como al método de su evaluación utilizado, dado que la metodología de evaluación por la adquisición de competencias y la metodología de enseñanza-aprendizaje no están totalmente implantadas en la docencia universitaria Gimeno, (2008). Esta es la razón por la que se ha se ha realizado este estudio, que ha surgido tras identificar la necesidad de desarrollar un proyecto de innovación docente dedicado a definir y poner en práctica acciones formativas que mejoren la evaluación universitaria por competencias.

\title{
2. Antecedentes
}

Los antecedentes de este estudio-proyecto están anclados en el antiguo sistema de evaluación del Plan de Estudios Universitarios de 1997, según el cual se han venido evaluando a los estudiantes de las asignaturas de escultura de la Licenciatura en Bellas Artes valorando en ellos la adquisición de conocimientos teóricos, las habilidades técnicas desarrolladas, su actitud y asistencia a clase, su capacidad de integración en el grupo y el nivel de habilidades propias de exposición y defensa de sus trabajos y, por último, el resultado final de los ejercicios escultóricos realizados. 
Para conseguir con éxito el cambio que supone la adaptación del anterior sistema docente al nuevo EEES en la titulación de Bellas Artes, es necesaria una renovación importante en el sistema de evaluación-calificación. La evaluación es un componente esencial en el proceso de enseñanza-aprendizaje en el marco de la convergencia europea, que regula y valora el nivel de conocimientos y destrezas adquiridas por el alumno, y también la capacidad del profesor para transmitir los conocimientos y generar formación y conocimiento en el alumno. Pero a la vez, no olvida la adquisición de competencias por el estudiante que le doten de capacidades formativas y humanas, capaces de hacer de él una persona competitiva en el ámbito académico y laboral (Rodríguez e Ibarra, 2011).

La evaluación no sólo debe contemplar una calificación final, sino que ha de ser una herramienta que permita al estudiante evaluar su propio proceso de aprendizaje $\mathrm{y}$, que a la vez ayude al docente a mejorar su actividad profesional, mediante la autoevaluación o la evaluación que de él hagan los estudiantes. debe desarrollarse en varios momentos del periodo formativo: evaluación inicial, continua y final. Debe ser sumativa y formativa. Por tanto, la evaluación es un proceso complejo que debe ser interpretado como un elemento que permita obtener información para mejorar el proceso educativo.

Hoy en día la evaluación está favorecida por el uso las TIC (e-Evaluación), que proporciona el diseño de diferentes formas de evaluación. Estas técnicas han de ser un medio que permita una aproximación entre los alumnos y el docente en el escenario virtual educativo y no ser exclusivamente un fin en sí mismas para la evaluación, sino que deben sumarse a la evaluación presencial, siempre que sea posible compatibilizar ambas posibilidades (Rodríguez e Ibarra, 2011).

\section{Objetivos}

En este trabajo se ha formulado un objetivo general, que ha consistido en estudiar varias competencias específicas de la asignatura Escultura III: Metodologías escultóricas, pertenecientes al Grado en Bellas Artes (Universidad de Granada), que figuran en su Guía Docente (UGR, 2012), teniendo en cuenta las competencias generales del texto Verificado del Grado en Bellas Artes (UGR, 2010) que están directamente relacionadas con las específicas elegidas. Además, se ha propuesto redefinir dichas competencias y diseñar una programación docente adecuada para poderlas trabajar con los estudiantes de la asignatura y evaluar en ellos la adquisición de dichas competencias, así como el proyecto docente desarrollado.

Para alcanzar el este objetivo general se han desarrollado varios objetivos secundarios, que han sido:

- Seleccionar adecuadamente las competencias generales y específicas a trabajar. 
- Identificar las subunidades de competencias específicas (desagregarlas) y redefinirlas.

- Diseñar y programar actividades docentes dirigidas al alumnado, enfocadas a la adquisición de las redefinidas subunidades de competencias.

- Diseñar un sistema de evaluación por competencias que permita conocer el nivel de adquisición de las mismas por el estudiante, cuyo resultado quede reflejado en la calificación final obtenida por él en la asignatura.

- Poner en práctica el modelo educativo diseñado, exponer sus resultados, evaluarlo y determinar su utilidad.

\section{Metodología}

El sistema de trabajo realizado ha desarrollado una metodología activa que ha favorecido que el alumnado y el profesorado implicados impulsen y desarrollen el proceso de enseñanza-aprendizaje. Se ha tratado de una metodología compartida y actualizada, acorde con las demandas formativas de los estudiantes universitarios que se preparan para responder adecuadamente a la demanda del mercado laboral y satisfacer sus propios intereses profesionales, (Jimeno et al., 2008), tal como es inmanente al nuevo título académico del Grado en Bellas Artes (Universidad de Granada, 2010).

La metodología usada se ha dividido en fases, para seguir consecutivamente el proceso adecuado de trabajo.

\section{$1^{\text {a }}$ FASE}

El punto de partida de la metodología ha sido el diseño, planificación y presentación de un proyecto de innovación docente, basado en programar actividades concretas que ayuden a mejorar la docencia. Dicho proyecto ha estado basado en la planificación y evaluación por competencias de la actividad docente, siguiendo las indicaciones recogidas en los documentos oficiales del texto Verificado del Grado en Bellas Artes y en la Guía Docente de la asignatura, que parten del Libro Blanco del Grado en Bellas Artes, Diseño y Restauración (ANECA, 2004), ante la necesidad de establecer este nuevo sistema formativo universitario (Villa, Poblet, García et al., 2008).

Para elaborar el proyecto de innovación docentes, en primer lugar, se han elegido las competencias específicas de la asignatura y las generales del Grado sobre las que trabajar de entre las que figuran en su Guía Docente (UGR, 2012), poniendo especial cuidado en seleccionar aquellas que están directamente relacionadas con la asignatura.

Después, se ha pasado al estudio detallado de las competencias seleccionadas, a la desagregación de las mismas y a la redefinición por separado de cada una de sus unidades de competencia, así como a la redacción del plan de innovación docente, 
en función de la información obtenida. Este proyecto ha incluido la programación, metodología y su propuesta de evaluación de la asignatura.

\section{$2^{\mathrm{a}}$ FASE}

Esta parte ha consistido en la presentación del proyecto de innovación a los estudiantes, durante los primeros días de clase de la asignatura, habiendo sido bien acogido por ellos. La implantación de dicho proyecto ha tenido lugar desde el principio de la docencia y ha sido continua y progresiva, pretendiendo el desarrollo del proceso educativo necesario para la adquisición de las competencias referentes a la asignatura y, especialmente, las seleccionadas.

\section{$3^{\mathrm{a}}$ FASE}

La tercera fase se ha dedicado a la evaluación de las diferentes acciones puestas en práctica con esta acción formativa. Ha contado con diversas herramientas de evaluación, en forma de encuestas y formularios referentes a la evaluación del profesorado, la autoevaluación (profesores y estudiantes) y la coevaluación del alumnado.

La evaluación se ha realizado según una temporalización continuada, que ha contado con una evaluación inicial, una continua, desarrollada en el transcurso del curso, y una final. Cada una de ellas ha tenido diferentes formularios.

La primera evaluación se ha dedicado a conocer el nivel inicial formativo que han presentado los estudiantes de cada grupo y a detectar sus principales deficiencias formativas.

La segunda etapa evaluativa ha consistido en evaluaciones continuas hechas por los alumnos y el profesorado, en las que se han puesto en práctica la autoevaluación del propio alumno, del profesor y la coevaluación por parte de grupo o evaluación entre iguales.

En la tercera etapa el estudiante ha realizado una auto-evaluación final de su propio aprendizaje y de su adquisición de competencias. También, se ha llevado a cabo una evaluación hecha por el alumnado sobre el proyecto de innovación docente desarrollado (contenidos, metodología, resultados esperados, adecuación al nivel formativo, etc.).

Así mismo, el profesorado implicado ha realizado una autoevaluación sobre la calidad del proceso de enseñanza-aprendizaje desarrollada, la adecuación de las actividades propuestas, la programación diseñada, la metodología seguida, la evaluación aplicada y demás aspectos que han conllevado la puesta en práctica de esta mejora docente.

Además, se ha contado con una evaluación externa hecha por el propio Departamento de Escultura, en la que han participado los técnicos de taller implicados en la 
docencia y el profesorado relacionado con la materia, pero que no imparte está misma asignatura.

\section{$4^{\mathrm{a}}$ FASE}

La cuarta fase ha estado dedicada a la recogida de datos de evaluación, al análisis de los mismos y a la discusión de los resultados obtenidos. Finalmente, el profesorado implicado ha emitido las calificaciones que los estudiantes han obtenido en la asignatura, evaluando su adquisición de las competencias trabajadas, en función de las evaluaciones obtenidas sobre ellos y los criterios generales de evaluación que figuran en la Guía Docente de la asignatura.

Además, esta última fase ha incluido la elaboración de una Memoria Final del proyecto y la elaboración de recursos didácticos generados por el mismo.

\section{Cronograma}

Partiendo de que se ha tratado de una asignatura de carácter semestral $\left(4^{\circ}\right.$ semestre de la titulación) de $2^{\circ}$ curso del Grado, temporalizada entre los meses de febrero y julio/septiembre de 2013, su desarrollo ha transcurrido según el siguiente cronograma: $1^{\mathrm{a}}$ Fase (meses de octubre de 2012 a febrero 2013), $2^{\mathrm{a}}$ Fase (meses de febrero a junio de 2013), $3^{\mathrm{a}}$ Fase (mes de junio 2013) y $4^{\mathrm{a}}$ Fase (meses de julio y septiembre 2013)

\section{Resultados}

Las competencias seleccionadas del texto Verificado del Grado en Bellas Artes (UGR, 2010) han sido (Tabla 1): 
Tabla 1: Exposición de las competencias generales seleccionadas, resultados de aprendizaje expresados en el texto del Grado en Bellas Artes, resultados declarados en la Guía Docente de la asignatura y reformulación de las competencias

\begin{tabular}{|c|c|c|c|}
\hline $\begin{array}{l}\text { Competencias } \\
\text { generales } \\
\text { (Grado en Bellas } \\
\text { Artes) }\end{array}$ & $\begin{array}{l}\text { Resultados de aprendizaje } \\
\text { relacionados y declarados en } \\
\text { el Grado en Bellas Artes }\end{array}$ & $\begin{array}{l}\text { Resultados de } \\
\text { aprendizaje } \\
\text { declarados en la } \\
\text { Guía Docente de la } \\
\text { asignatura tratada }\end{array}$ & $\begin{array}{l}\text { Reformulación } \\
\text { de los resultados } \\
\text { de aprendizaje } \\
\text { por competencia } \\
\text { derivados de la Guía } \\
\text { Docente }\end{array}$ \\
\hline $\begin{array}{l}\text { CE6. } \\
\text { Conocimiento } \\
\text { del vocabulario, } \\
\text { códigos, y } \\
\text { conceptos } \\
\text { inherentes } \\
\text { al ámbito artístico }\end{array}$ & $\begin{array}{l}\text { Adquirir la habilidad para } \\
\text { elaborar estrategias de creación } \\
\text { artística mediante la realización } \\
\text { de proyectos individuales o en } \\
\text { equipo, bajo la conciencia de la } \\
\text { capacidad transformadora del } \\
\text { arte, como agente activo en la } \\
\text { configuración de las esculturas }\end{array}$ & $\begin{array}{l}\text { Comprensión y } \\
\text { aplicación artística } \\
\text { de los fundamentos y } \\
\text { conceptos inherentes } \\
\text { a cada técnica }\end{array}$ & $\begin{array}{l}\text { Capacidad de } \\
\text { comprender los } \\
\text { lenguajes artísticos } \\
\text { escultóricos, mediante } \\
\text { la terminología } \\
\text { propia de la materia } \\
\text { (vocabulario) y los } \\
\text { conceptos inherentes } \\
\text { a cada materia } \\
\text { escultórica }\end{array}$ \\
\hline $\begin{array}{l}\text { CE10. } \\
\text { Conocimiento } \\
\text { de los } \\
\text { materiales y de } \\
\text { sus } \\
\text { procesos } \\
\text { derivados } \\
\text { de creación y/o } \\
\text { producción }\end{array}$ & $\begin{array}{l}\text { Dotar a los estudiantes de los } \\
\text { instrumentos necesarios para la } \\
\text { integración de sus } \\
\text { conocimientos en procesos } \\
\text { de creación autónoma y/o de } \\
\text { experimentación interdisciplinar } \\
\text { para que puedan desarrollar su } \\
\text { práctica artística en todo tipo de } \\
\text { formatos y espacios culturales }\end{array}$ & $\begin{array}{l}\text { Conocimiento de } \\
\text { métodos y técnicas de } \\
\text { producción asociados } \\
\text { a los lenguajes } \\
\text { artísticos }\end{array}$ & $\begin{array}{l}\text { Aplicación de los } \\
\text { métodos y técnicas de } \\
\text { producción artística } \\
\text { a su propia creación } \\
\text { personal autónoma en } \\
\text { la escultura }\end{array}$ \\
\hline $\begin{array}{l}\text { CE9. } \\
\text { Conocimiento } \\
\text { de métodos } \\
\text { y técnicas de } \\
\text { producción } \\
\text { asociados a } \\
\text { los lenguajes } \\
\text { artísticos }\end{array}$ & $\begin{array}{l}\text { Adquirir la capacidad de } \\
\text { desarrollar procesos de creación } \\
\text { artística mediante el aprendizaje } \\
\text { de las diferentes tecnologías }\end{array}$ & $\begin{array}{l}\text { Conocimiento de } \\
\text { los instrumentos } \\
\text { y métodos de } \\
\text { experimentación en } \\
\text { arte }\end{array}$ & $\begin{array}{l}\text { Capacidad de utilizar } \\
\text { los instrumentos } \\
\text { y los métodos de } \\
\text { experimentación que } \\
\text { le permitan su propia } \\
\text { creación artística }\end{array}$ \\
\hline
\end{tabular}

Fuente: UGR y la autora.

Competencia 1": "CE6. Conocimiento del vocabulario, códigos, y conceptos inherentes al ámbito artístico".

Competencia $2^{\text {a. }}$ CE10. "Conocimiento de los materiales y de sus procesos derivados de creación y/o producción”.

Competencia $3^{\text {a }}$ : CE9. “Conocimiento de métodos y técnicas de producción asociados a los lenguajes artísticos”. 
Las competencias específicas de la Guía Docente de la asignatura (UGR, 2012), relacionadas respectivamente con las generales, son las siguientes:

1. "Conocimiento del vocabulario, códigos y conceptos inherentes a la creación escultórica".

2. "Conocimiento de los materiales y de sus procesos derivados de la creación y/ o producción escultórica”.

3. "Conocimiento de los métodos y técnicas de producción asociados a los lenguajes de carácter tridimensional".

\subsection{Estudio de las competencias}

\section{Competencia $1^{\text {a }}$ : CE6}

"Conocimiento del vocabulario, códigos, y conceptos inherentes al ámbito artístico" (UGR, 2012).

Unidades de competencias: conocer y utilizar adecuadamente el vocabulario específico de la escultura, conocer los códigos artísticos que definen la escultura; conocer los conceptos específicos de la escultura: concepto, volumen, espacio, materia, forma, técnica, mensaje artístico.

Metodología de enseñanza: lección magistral y análisis de casos.

Actividades: lecturas, realización de ejercicios de descripción de obras artísticas, presentación, corrección de trabajos y evaluación.

Lugar de ejecución: Aula Teórica de Escultura III, Biblioteca de UGR y Aula de Informática.

Tipo de trabajo: realizado por grupos de cinco estudiantes.

\section{Competencia $2^{\mathrm{a}}$ : CE10}

"Conocimiento de los materiales y de sus procesos derivados de creación y/o producción" (UGR, 2012).

Unidades de competencias: conocimiento de la madera como material escultórico y su proceso de producción; conocimiento de la piedra como material escultórico y su proceso de producción; conocimiento del metal como material escultórico y su proceso de producción; conocimiento de nuevos materiales escultórico y su proceso de producción; conocimiento de materiales alternativos escultóricos y su proceso de producción.

Metodología de enseñanza: resolución de ejercicios y problemas. 
Lugar de ejecución: Talleres de Escultura (metal, piedra, madera y vaciado) y Aula Teórica de Escultura III.

Actividades: realización de ejercicios prácticos en piedra, madera, metal o nuevos materiales por el estudiante, junto a la presentación, defensa, corrección y evaluación de los trabajos realizados.

Tipo de trabajo: Individual del estudiante.

\section{Competencia $3^{\text {a }}$ : CE9}

"Conocimiento de métodos y técnicas de producción asociados a los lenguajes artísticos" (UGR, 2012).

Unidades de competencia: conocimiento de los métodos aditivos: modelado; conocimiento de los métodos sustractivos: talla en piedra y madera; conocimiento de los métodos constructivos: ensamblaje, soldadura, atornillado, encolado, anudado, tejido y cosido; conocimiento de métodos mixtos: construcción de moldes, vaciado y fundición de metales.

Metodología de enseñanza: resolución de ejercicios y problemas.

Actividades: realización de ejercicios prácticos en piedra, madera, metal o nuevos materiales, junto a la presentación, defensa, corrección y evaluación de los trabajos presentados.

Lugar de ejecución: Talleres de Escultura (metal, piedra, madera y vaciado) y Aula Teórica de Escultura III.

Tipo de trabajo: Individual del estudiante.

\subsection{Evaluación de las competencias}

\subsubsection{Construcción de técnicas e instrumentos para la evaluación de la adquisición} de competencias y elaboración del sistema de calificación

Para evaluar adecuadamente una competencia se han de tener en cuenta los conocimientos implementados, las actitudes desarrolladas y las destrezas adquiridas. Los criterios de evaluación han sido los que figuran en la Guía Docente de la asignatura (UGR, 2012). Véanse:

"Nivel de adecuación, riqueza y rigor en los procesos y grado de concreción del trabajo, tanto práctico como teórico, individual o de grupo.

Capacidad de relación, reflexión, crítica y de autoevaluación en las distintas actividades. 
Nivel de interés, asistencia, constancia y puntualidad en el desarrollo y conclusión de las actividades propuestas. Este grado de interés contempla todas las acciones formativas.

Destreza, creatividad y originalidad en el desarrollo de prácticas y la elaboración de proyectos memoria.

Habilidad para la presentación y exposición de los resultados de su aprendizaje”.

\subsubsection{Procedimiento de evaluación}

El procedimiento de evaluación ha contemplado el seguimiento realizado por el estudiante del planteamiento, contenido, proceso educativo y demás iniciativas educativas propuestas por el profesor a nivel individual o de grupo (Tabla 2).

Tabla 2: Porcentaje de evaluación aplicada en la calificación del alumno, según consta en la Guía Docente de la asignatura. Fuente: UGR, 2012

Evaluación del grado de adquisición de competencias relacionadas con los contenidos de la materia a través de la evaluación continuada de las prácticas de creación, y trabajos propuestos mediante el seguimiento en el Aula Taller.

Resultado del estudio, lecturas, redacción y exposición de trabajos, cuadernos de campo, visitas de exposiciones, museos y ferias de arte Asistencia y participación activa en las clases, seminarios y tutorías académicas.

Entre el 50 a $60 \%$

Entre el 30 a $40 \%$

Hasta $10 \%$

También ha incluido la evaluación de las producciones procedentes del trabajo individual o grupal del alumnado su presentación ante los otros estudiantes y el profesor. Además, ha comprendido la autoevaluación de sí mismo, la coevaluación, la evaluación del proceso de enseñanza y la evaluación de la práctica docente.

El porcentaje de calificación aplicado a la nota final del alumnado se ha ajustado a lo establecido en la Guía Docente de la asignatura (UGR, 2012).

\subsubsection{Resultados de evaluación}

Los resultados del aprendizaje de los estudiantes han contemplado los conocimientos adquiridos, los productos elaborados (producciones), las actitudes desarrolladas y las destrezas aprendidas. Con todo ello, los resultados han sido positivos, según se deriva de las calificaciones obtenidas por los estudiantes de los Grupo B y D implicados, como se observa en los gráficos presentados (Figs. 1 A y B, respectivamente). 
Figura 1: A. Resultados de las calificaciones obtenidas por los estudiantes del Grupo B en la asignatura Escultura III: Metodologías escultóricas, expresadas en estadísticas porcentuales. B. Resultados de las calificaciones obtenidas por los alumnos del Grupo D en la asignatura Escultura III: Metodologías escultóricas, expresadas en estadísticas porcentuales
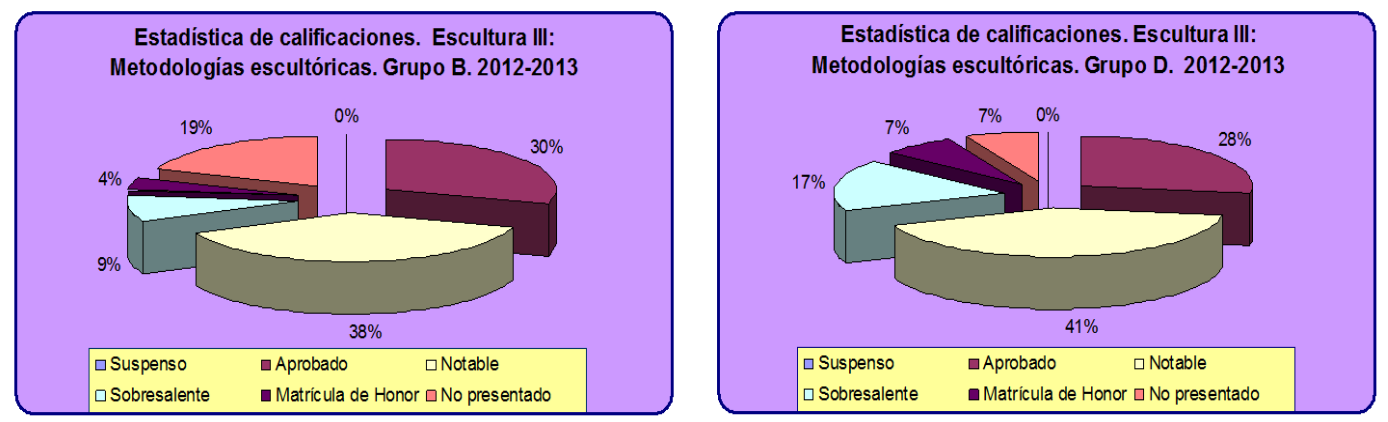

Comparando los gráficos expuestos (Fig. 1. A y B), las calificaciones obtenidas han sido mejores en el Grupo D (horario de tarde, 29 alumnos), que en el grupo B (horario de mañana, 47 alumnos). Esto puede haberse debido a que se ha tratado de un conjunto de alumnos/as menos numeroso, lo que ha favorecido el desarrollo del trabajo.

Figura 2: A. Estadísticas porcentuales de superación global de la asignatura Escultura III: Metodologías escultóricas, referentes al Grupo B. B. Estadísticas porcentuales de superación global de la asignatura Escultura III: Metodologías escultóricas, referentes al Grupo D.
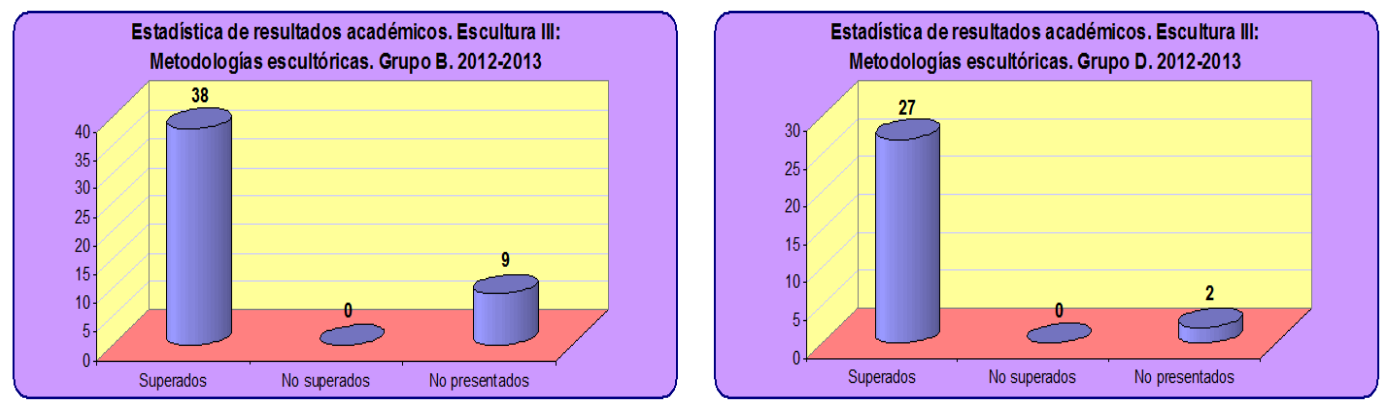

El Grupo B (Fig. 1 A) ha obtenido unas calificaciones de 0 Suspensos, 14 Aprobados, 18 Notables, 4 Sobresalientes, 2 Matrículas de Honor y 9 No Presentados, contando con un total de 49 alumnos. Sus resultados globales de calificaciones han sido (Fig. 2 A): 38 Aprobados, 0 Suspensos y 9 No Presentados

El Grupo D (Fig. 1B) ha obtenido unas calificaciones de 0 Suspensos, 8 Aprobados, 12 Notables, 5 Sobresaliente, 2 Matrículas de Honor y 2 No Presentados. Sus resultados globales de calificaciones han sido (Fig. 2 B).: 27 Aprobados, 0 Suspensos y 2 No Presentados. 
Como resultados finales, se ha diseñado una Guía de Trabajos a realizar, que será complementaria a la Guía Docente (UGR, 2012) de la asignatura. Se han diseñado y utilizado instrumentos de evaluación propios, como encuestas y cuestionarios, que quedan disponibles al uso del profesorado interesado. Se ha elaborado una Memoria Final del proyecto elaborado y puesto en práctica, que recoge todas las características del mismo, contemplando la metodología, las herramientas y modelo de evaluación, además de los resultados obtenidos.

\section{Conclusiones}

Partiendo de los objetivos planteados y atendiendo a los resultados logrados, se ha comprobado que la posibilidad de desarrollar convenientemente las competencias elegidas, con el diseño de la programación de la asignatura, una metodología apropiada y la aplicación de la evaluación del alumno por competencias.

La adecuada segregación de las competencias específicas y generales elegidas ser trabajardas y su redefinición permiten abordarlas de manera progresiva y eficiente durante el proceso formativo.

La actividad de innovación docente, basada en el diseño y puesta en práctica de este sistema evaluativo por competencias, ha dado unos resultados docentes buenos y esto ha quedado reflejado en las calificaciones obtenidas por los alumnos/as de la asignatura.

El alumnado se ha implicado en el proceso educativo, lo que ha favorecido el proceso de enseñanza-aprendizaje y ha beneficiado a la titulación del Grado en Bellas Artes.

Por último, el proyecto de innovación docente ha sido bien evaluado, lo que ha permitido pensar que la evaluación por competencias en el Grado en Bellas Artes de la Universidad de Granada continuará su implantación en lo sucesivo.

\section{Referencias bibliográficas}

Libros

ÁLVAREZ ROJO, Víctor (2010). Evaluación de competencias en la Universidad. ¿Por qué cambiar el modelo de evaluación tradicional? Sevilla: Universidad de Sevilla.

GIMENO SACRISTÁN, José; (et al.) (2008). Educar por competencias, ¿qué hay de nuevo? Madrid: Morata. 
RODRÍGUEZ GÓMEZ, Gregorio e IBARRA SÁIZ, María Soledad (Eds.) (2011). e-Evaluación orientada al e-Aprendizaje estratégico en la Educación Superior. Madrid: Nancea.

VILLA SÁNCHEZ, Aurelio; POBLETE RUÍZ, Manuel (Dirs.); GARCÍA OLALLA, Ana et al. (Equipo de investigación) (2008). Aprendizaje basado en competencias: una propuesta para la evaluación de las competencias genéricas. Bilbao: Universidad de Deusto.

Artículos de Revistas en papel

IBARRA SÁIZ, María Soledad y RODRÍGUEZ GÓMEZ, Gregorio. (2010). “Aproximación al discurso dominante sobre la evaluación del aprendizaje en la universidad”. En Revista de Educación, (351), 385-407.

Documentos y artículos en publicaciones web

ANECA (2004). Libro Blanco del Grado en Bellas Artes, Diseño y Restauración. Disponible en: http://www.aneca.es/var/media/150332/libroblanco_bellasartes def.pdf. [Consulta: 02-09-2012].

ANECA (2012, última actualización). Guía de Apoyo para la elaboración de las Memorias de Verificación de Títulos Oficiales Universitarios (Grado y Master). Disponible en: http://www.aneca.es/content/download/12155/136031/file/verifica_guia_v04_120116.pdf. [Consulta: 03- 09-2013].

UGR, Universidad de Granada (2010). Documento Verifica (Grado Verificado). Titulo: Grado en Bellas Artes. Disponible en: http://www.bellasartesgranada.org/ index.php/Asignaturas-Guias-Docentes/474/0/RIFICADO.pdf. [Consulta: 10-092012].

UGR, Universidad de Granda (2012). Guía Docente de la asignatura: Escultura III: Metodologías escultóricas, curso académico 2012-2013. Disponible en: http:// www.bellasartesgranada.org/index.php/Asignaturas-Guias-Docentes/474/0/RIFICADO.pdf. [Consulta: 18-09-2012]. 